\title{
A New Minkowski Distance Based on Induced Aggregation Operators
}

\author{
José M. Merigó, Montserrat Casanovas \\ Department of Business Administration, University of Barcelona, \\ Av. Diagonal 690, Barcelona, 08034, Spain \\ E-mail: jmerigo@ub.edu,mcasanovas@ub.edu \\ Received: 25-05-2009 \\ Accepted: 01-12-2010
}

\begin{abstract}
The Minkowski distance is a distance measure that generalizes a wide range of distances such as the Hamming and the Euclidean distance. In this paper, we develop a generalization of the Minkowski distance by using the induced ordered weighted averaging (IOWA) operator. We call it the induced Minkowski OWA distance (IMOWAD) or induced generalized OWA distance (IGOWAD) operator. Then, we are able to obtain a wide range of distance measures that includes the Minkowski distance, the Minkowski OWA distance (MOWAD), and the induced OWA distance (IOWAD). We also present a further generalization by using quasi-arithmetic means. We end the paper with a numerical example of the new approach.
\end{abstract}

Keywords: Minkowski distance, Aggregation operators, IOWA operator, Decision making.

\section{Introduction}

The Minkowski distance is one of the main distance measures because it generalizes a wide range of other distances such as the Hamming distance and the Euclidean distance. Often, when calculating distances, we want an average result of all the individual distances. We call this the normalization process. In the literature, we find mainly three types of normalized distances. The first one is when we use the arithmetic mean and it is known as the normalized Minkowski distance (NMD). The second one is when we use the weighted average (WA) and it is known as the weighted Minkowski distance (WMD). The third one is when we use the ordered weighted averaging (OWA) operator ${ }^{1-24}$ and it is known as the Minkowski ordered weighted averaging distance (MOWAD) operator. ${ }^{8,11}$ Note that the MOWAD includes the NMD and the WMD as special cases.
Sometimes, when normalizing the Minkowski distance with the OWA operator, it would be interesting to consider a more general formulation of the reordering process. A very useful technique for doing so is the induced OWA (IOWA) operator. $^{17,21}$ The IOWA operator provides a parameterized family of aggregation operators such as the maximum, the minimum, the average and the OWA operator. Thus, we are able to use complex reordering processes in the aggregation step of the IOWA operator. This can be useful in a lot of situations such as in decision making problems ${ }^{25-28}$ where we may consider complex attitudinal characters of the decision maker instead of simply considering the degree of optimism or pessimism. Recently, Merigó and Gil-Lafuente $^{12}$ suggested a more general formulation of the IOWA operator by using generalized and quasiarithmetic means. They called these new aggregation operators, the induced generalized OWA (IGOWA) operator and the induced Quasi-OWA (Quasi-IOWA) operator. They generalize a wide range of aggregation 
operators including the IOWA operator, the OWA operator, the average and the weighted average.

In this paper, we suggest a new type of distance measure consisting in normalizing the Minkowski distance by using the IOWA operator. Then, the normalization developed will be able to reflect complex attitudinal characters. We will call this generalization as the induced Minkowski OWA distance (IMOWAD) operator. The main advantage of this operator is that it generalizes a wide range of distances such as the NMD, the WMD, the MOWAD, the induced OWA distance (IOWAD), the induced Euclidean OWA distance (IEOWAD) and a lot of other particular cases. Another advantage of the IMOWAD operator is that it is able to deal with complex attitudinal characters (or complex degrees of orness) in the aggregation process. Therefore, we are able to deal with more complex situations more close to the real world.

We further generalize the IMOWAD operator by using quasi-arithmetic means. As a result, we get the Quasi-IOWAD operator. It is a more complete generalization because it includes the IMOWAD operator and a lot of other situations. We also develop an application of the new approach in a decision making problem about selection of investments. We see that depending on the particular type of IMOWAD operator used, the results may lead to different decisions.

This paper is organized as follows. In Section 2, we briefly review some basic concepts about the Minkowski distance, the IOWA and the IGOWA operator. In Section 3 we present the IMOWAD operator. Section 4 analyzes different families of IMOWAD operators. In Section 5 we present the QuasiIOWAD operator. Section 6 analyzes the applicability of the new approach and Section 7 develops a numerical example of the new generalization. Finally, in Section 8 we summarize the main conclusions of the paper.

\section{Preliminaries}

In this Section, we briefly review the Minkowski distance, the IOWA operator and the IGOWA operator.

\subsection{Normalized Minkowski Distance}

The normalized Minkowski distance is a distance measure ${ }^{29-34}$ that generalizes a wide range of distances such as the normalized Hamming distance and the normalized Euclidean distance. In fuzzy set theory, it can be useful, for example, for the calculation of distances between fuzzy sets, interval-valued fuzzy sets, intuitionistic fuzzy sets, etc.

In order to define the Minkowski distance, first, we define a distance measure. Essentially, a distance measure has to accomplish the following properties.

1) Non-negativity: $\mathrm{D}\left(A_{1}, A_{2}\right) \geq 0$.

2) Commutativity: $\mathrm{D}\left(A_{1}, A_{2}\right)=\mathrm{D}\left(A_{2}, A_{1}\right)$.

3) Reflexivity: $\mathrm{D}\left(A_{1}, A_{1}\right)=0$.

4) Triangle inequality: $\mathrm{D}\left(A_{1}, A_{2}\right)+\mathrm{D}\left(A_{2}, A_{3}\right) \geq$ $\mathrm{D}\left(A_{1}, A_{3}\right)$.

The normalized Minkowski distance can be formulated for two sets $A$ and $B$ as follows.

Definition 1. A normalized Minkowski distance of dimension $n$ is a mapping $d_{m}: R^{n} \times R^{n} \rightarrow R$ such that:

$$
d_{m}(A, B)=\left(\frac{1}{n} \sum_{i=1}^{n}\left|a_{i}-b_{i}\right|^{\lambda}\right)^{1 / \lambda}
$$

where $a_{i}$ and $b_{i}$ are the ith arguments of the sets $A$ and $B$ and $\lambda$ is a parameter such that $\lambda \in(-\infty, \infty)$.

If we give different values to the parameter $\lambda$, we can obtain a wide range of special cases. For example, if $\lambda=1$, we obtain the normalized Hamming distance (NHD). If $\lambda=2$, the normalized Euclidean distance (NED).

Sometimes, when normalizing the Minkowski distance, we prefer to give different weights to each individual distance. Thus, the distance is known as the weighted Minkowski distance. It can be defined as follows.

Definition 2. A weighted Minkowski distance of dimension $n$ is a mapping $d_{w m}: R^{n} \times R^{n} \rightarrow R$ that has an associated weighting vector $W$ of dimension $n$ with $\sum_{j=1}^{n} w_{j}=1$ and $w_{j} \in[0,1]$, such that:

$$
d_{w m}(A, B)=\left(\sum_{i=1}^{n} w_{i}\left|a_{i}-b_{i}\right|^{\lambda}\right)^{1 / \lambda}
$$

where $a_{i}$ and $b_{i}$ are the ith arguments of the sets $A$ and $B$ and $\lambda$ is a parameter such that $\lambda \in(-\infty, \infty)$.

In this case, we can also obtain a wide range of special cases by using different values in the parameter $\lambda$. For example, if $\lambda=1$, we obtain the weighted 
Hamming distance (WHD). If $\lambda=2$, the weighted Euclidean distance (WED).

\subsection{Induced OWA Operator}

The IOWA operator was introduced by Yager and Filev $^{21}$ and it is an extension of the OWA operator. Its main difference is that the reordering step is not developed with the values of the arguments. In this case, the reordering step is developed with order inducing variables. It can be defined as follows.

Definition 3. An IOWA operator of dimension $n$ is a mapping $f: R^{n} \rightarrow R$ that has an associated weighting vector $W$ of dimension $n$ with $\sum_{j=1}^{n} w_{j}=1$ and $w_{j} \in[0,1]$, such that:

$$
f\left(\left\langle u_{1}, a_{1}\right\rangle,\left\langle u_{2}, a_{2}\right\rangle, \ldots,\left\langle u_{n}, a_{n}\right\rangle\right)=\sum_{j=1}^{n} w_{j} b_{j}
$$

where $b_{j}$ is the $a_{i}$ value of the IOWA pair $\left\langle u_{i}, a_{i}\right\rangle$ having the jth largest $u_{i}, u_{i}$ is the order inducing variable and $a_{i}$ is the argument variable.

The IOWA operator includes the OWA operator as a particular case and a lot of other situations such as the maximum, the minimum and the average. Note that it is possible to distinguish between the descending IOWA (DIOWA) operator and the ascending IOWA (AIOWA) operator.

\subsection{Induced Generalized OWA Operator}

The IGOWA operator was introduced in Ref. 12 and it represents a generalization of the IOWA operator by using generalized means. Therefore, it is possible to include in the same formulation, different types of induced aggregation operators such as the IOWA operator or the induced OWG (IOWG) operator. It can be defined as follows.

Definition 4. An IGOWA operator of dimension $n$ is a mapping IGOWA: $R^{n} \times R^{n} \rightarrow R$ that has an associated weighting vector $W$ of dimension $n$ such that $w_{j} \in[0,1]$ and $\sum_{j=1}^{n} w_{j}=1$, then:

$$
f\left(\left\langle u_{1}, a_{1}\right\rangle, \ldots,\left\langle u_{n}, a_{n}\right\rangle\right)=\left(\sum_{j=1}^{n} w_{j} b_{j}^{\lambda}\right)^{1 / \lambda}
$$

where $b_{j}$ is the $a_{i}$ value of the IGOWA pair $\left\langle u_{i}, a_{i}\right\rangle$ having the jth largest $u_{i}, u_{i}$ is the order inducing variable, $a_{i}$ is the argument variable and $\lambda$ is a parameter such that $\lambda \in(-\infty, \infty)$.

As we can see, if $\lambda=1$, we get the IOWA operator. If $\lambda=0$, the IOWG operator and if $\lambda=2$, the IOWQA operator. Note that it is possible to further generalize the IGOWA operator by using quasi-arithmetic means. The result is the Quasi-IOWA operator. For further reading on the IGOWA and the Quasi-IOWA, refer to Ref. 12.

\section{The Induced Minkowski OWA Distance}

The IMOWAD operator is a distance measure that uses the IOWA operator in the normalization process of the Minkowski distance. Then, the reordering of the individual distances is developed with order inducing variables. For two sets $X=\left\{x_{1}, \ldots, x_{n}\right\}$ and $Y=\left\{y_{1}, \ldots\right.$, $\left.y_{n}\right\}$, it can be defined as follows.

Definition 5. An IMOWAD operator is a mapping $f: R^{n}$ $\times R^{n} \times R^{n} \rightarrow R$ that has an associated weighting vector $W$ with $w_{j} \in[0,1]$ and $\sum_{j=1}^{n} w_{j}=1$, such that:

$$
f\left(\left\langle u_{1}, x_{1}, y_{1}\right\rangle, \ldots,\left\langle u_{n}, x_{n}, y_{n}\right\rangle\right)=\left(\sum_{j=1}^{n} w_{j} b_{j}^{\lambda}\right)^{1 / \lambda}
$$

where $b_{j}$ is the $\left|x_{i}-y_{i}\right|$ value of the IMOWAD triplet $\left\langle u_{i}\right.$, $\left.x_{i}, y_{\mathrm{i}}\right\rangle$ having the jth largest $u_{i}, u_{i}$ is the order inducing variable, $\left|x_{i}-y_{i}\right|$ is the argument variable represented in the form of individual distances and $\lambda$ is a parameter such that $\lambda \in(-\infty, \infty)$.

Example 1. Assume two sets $A=(0.2,0.4,0.7,0.3)$ and $B=(0.9,0.4,0.7,0.2)$, and $\lambda=1$. Assume that both sets have the same order-inducing variables $U=(6,8,3,7)$. And assume the following weighting vector $W=(0.3$, $0.3,0.2,0.2)$. Then, the IMOWAD can be calculated as follows.

$\underline{\text { IMOWAD triplets }}$

$$
\begin{gathered}
\langle 6,0.2,0.9\rangle=\langle 6,0.7\rangle \\
\langle 8,0.4,0.4\rangle=\langle 8,0\rangle \\
\langle 3,0.7,0.7\rangle=\langle 3,0\rangle \\
\langle 7,0.3,0.2\rangle=\langle 7,0.1\rangle
\end{gathered}
$$

$$
f=0.3 \times 0+0.3 \times 0.1+0.2 \times 0.7+0.2 \times 0=0.17 \text {. }
$$

Note that sometimes, as in fuzzy set theory, it is better to use in the definition, a mapping $f:[0,1]^{n} \times[0$, $1]^{n} \rightarrow[0,1]$. 
A further interesting aspect to consider is the reordering process of the information. ${ }^{35}$ Usually, we reorder the IMOWAD according to the values of the $u_{i}$, but it is also possible to adapt them to the initial positions of the arguments. That is:

$$
f\left(\left\langle u_{1}, a_{1}\right\rangle,\left\langle u_{2}, a_{2}\right\rangle \ldots,\left\langle u_{n}, a_{n}\right\rangle\right)=\left(\sum_{i=1}^{n}\left|x_{i}-y_{i}\right|^{\lambda} w_{i}\right)^{1 / \lambda}
$$

where $w_{i}$ is the $i$ th weight $w_{j}$ reordered according to the positions of the $\left|x_{i}-y_{i}\right|$ and using order-inducing variables $u_{i}$.

The IOWAD operator is commutative, monotonic, bounded and idempotent. These properties can be proved with the following theorems.

Theorem 1 (Commutativity). Assume $f$ is the IMOWAD operator, then:

$$
\begin{aligned}
f\left(\left\langle u_{1}, x_{1}, y_{1}\right\rangle, \ldots,\right. & \left.\left\langle u_{n}, x_{n}, y_{n}\right\rangle\right)= \\
& =f\left(\left\langle u_{1}, c_{1}, d_{1}\right\rangle, \ldots,\left\langle u_{n}, c_{n}, d_{n}\right\rangle\right.
\end{aligned}
$$

where $\left(\left\langle u_{1}, x_{1}, y_{1}\right\rangle, \ldots,\left\langle u_{n}, x_{n}, y_{n}\right\rangle\right)$ is any permutation of the arguments $\left(\left\langle u_{1}, c_{1}, d_{1}\right\rangle, \ldots,\left\langle u_{n}, c_{n}, d_{n}\right\rangle\right)$.

Proof. It is straightforward and thus omitted.

Theorem 2 (Monotonicity). Assume $f$ is the IMOWAD operator, if $\left|x_{i}-y_{i}\right| \geq\left|c_{i}-d_{i}\right|$, for all $i$, then:

$$
\begin{aligned}
f\left(\left\langle u_{1}, x_{1}, y_{1}\right\rangle, \ldots,\left\langle u_{n}, x_{n}, y_{n}\right\rangle\right) & \geq \\
& \geq f\left(\left\langle u_{1}, c_{1}, d_{1}\right\rangle, \ldots,\left\langle u_{n}, c_{n}, d_{n}\right\rangle\right)
\end{aligned}
$$

Proof. It is straightforward and thus omitted.

Theorem 3 (Bounded). Assume $f$ is the IMOWAD operator, then:

$$
\begin{aligned}
& \min \left\{\left|x_{i}-y_{i}\right|\right\} \leq f\left(\left\langle u_{1}, x_{1}, y_{1}\right\rangle, \ldots,\left\langle u_{n}, x_{n}, y_{n}\right\rangle\right) \leq \\
& \leq \max \left\{\left|x_{i}-y_{i}\right|\right\}
\end{aligned}
$$

Proof. It is straightforward and thus omitted.

Theorem 4 (Idempotency). Assume $f$ is the IMOWAD operator, if $\left|x_{i}-y_{i}\right|=a$, for all $i$, then:

$$
f\left(\left\langle u_{1}, x_{1}, y_{1}\right\rangle, \ldots,\left\langle u_{n}, x_{n}, y_{n}\right\rangle\right)=a
$$

Proof. It is straightforward and thus omitted.
Remark 1. Note that if $x_{i}=y_{i}$ for all $i \in[1, n], f\left(\left\langle u_{1}, x_{1}\right.\right.$, $\left.\left.y_{1}\right\rangle, \ldots,\left\langle u_{n}, x_{n}, y_{n}\right\rangle\right)=0$. Note also that $f\left(\left\langle u_{1}, x_{1}, y_{1}\right\rangle, \ldots\right.$, $\left.\left\langle u_{n}, x_{n}, y_{n}\right\rangle\right)=f\left(\left\langle u_{1}, y_{1}, x_{1}\right\rangle, \ldots,\left\langle u_{n}, y_{n}, x_{n}\right\rangle\right)$.

Remark 2. Note that it is possible to distinguish between descending (DIMOWAD) and ascending (AIMOWAD) orders. The weights of these operators are related by $w_{j}=w^{*}{ }_{n+1-j}$, where $w_{j}$ is the $j$ th weight of the DIMOWAD (or IMOWAD) operator and $w_{n+1-j}{ }_{n+j}$ the $j$ th weight of the AIMOWAD operator.

Remark 3. If $B$ is a vector corresponding to the ordered arguments $b_{j}^{\lambda}$, we shall call this the ordered argument vector and $W^{T}$ is the transpose of the weighting vector, then, the IMOWAD operator can be expressed as:

$$
f\left(\left\langle u_{1}, x_{1}, y_{1}\right\rangle, \ldots,\left\langle u_{n}, x_{n}, y_{n}\right\rangle\right)=\left(W^{T} B\right)^{1 / \lambda}
$$

Remark 4. Note that if the weighting vector is not normalized, i.e., $W=\sum_{j=1}^{n} w_{j} \neq 1$, then, the IMOWAD operator can be expressed as:

$$
f\left(\left\langle u_{1}, x_{1}, y_{1}\right\rangle, \ldots,\left\langle u_{n}, x_{n}, y_{n}\right\rangle\right)=\left(\frac{1}{W} \sum_{j=1}^{n} w_{j} b_{j}^{\lambda}\right)^{1 / \lambda}
$$

Some other interesting generalizations can be developed following. ${ }^{36-39}$ Following, ${ }^{38}$ we can develop the function IMOWAD operator that uses a generating function $r$ for the order inducing variables such that, $r: I$ $\rightarrow R$, being $I \subset R$ a closed interval $I=[a, b]$. Moreover, we use a more general representation by using also a generating function for the arguments such that, $s: R^{m}$ $\rightarrow R$. Furthermore, we also use a weighting function $f$ for the weighting vector. Thus, we obtain the function induced generalized mixture distance (IGMD) operator as follows. Note that in this definition we refer to the arguments as two sets $X=\left\{x_{1}, x_{2}, \ldots, x_{n}\right\}$ and $Y=\left\{y_{1}\right.$, $\left.y_{2}, \ldots, y_{n}\right\}$.

Definition 6. An IGMD operator of dimension $n$ is a mapping IMD: $R^{n} \times R^{n} \times R^{n} \rightarrow R$ that has an associated a vector of weighting functions $f, r: I \rightarrow] 0, \infty[$, is some positive continuous function, $s: R^{m} \rightarrow R$, such that:

$\operatorname{IGMD}\left(\left\langle r_{o}\left(u_{1}\right), s_{p}\left(x_{1}\right), s_{q}\left(y_{1}\right)\right\rangle, \ldots,\left\langle r_{o}\left(u_{n}\right), s_{p}\left(x_{n}\right), s_{q}\left(y_{n}\right)\right\rangle\right)=$ 


$$
=\left(\frac{\sum_{j=1}^{n} f_{j}\left(s_{y}\left(b_{j}\right)\right) s_{y}\left(b_{j}\right)^{\lambda}}{\sum_{j=1}^{n} f_{j}\left(s_{y}\left(b_{j}\right)\right)}\right)^{1 / \lambda}
$$

where $s_{y}\left(b_{j}\right)$ is the $\left|s_{p}\left(x_{i}\right)-s_{q}\left(y_{i}\right)\right|$ value of the IGMD triplet $\left.\left\langle r_{o}\left(u_{i}\right), s_{p}\left(x_{i}\right), s_{q}\left(y_{i}\right)\right\rangle\right)$ having the jth largest $r_{o}\left(u_{i}\right)$, $u_{i}$ is the order-inducing variable, $\left|s_{p}\left(x_{i}\right)-s_{q}\left(y_{i}\right)\right|$ is the argument variable represented in the form of individual distances; $o, p$ and $q$ indicates that each order-inducing variable and each argument is formed by using a different function and $\lambda$ is a parameter such that $\lambda \in$ $(-\infty, \infty)$.

Following, ${ }^{12}$ we can obtain a wide range of particular cases of the IGMD operator. For example:

- If $\lambda=1$, we obtain the induced mixture distance (IMD) operator.

- If $\lambda=2$, the induced quadratic mixture distance (IQMD) operator.

- If $\lambda=3$, the induced cubic mixture distance (ICMD) operator.

- If $\lambda \rightarrow 0$, the induced geometric mixture distance operator

- If $\lambda=-1$, the induced harmonic mixture distance (IHMD) operator.

A further interesting extension consists in using infinitary aggregation operators. ${ }^{36}$ In this case, we assume that there are an unlimited number of arguments that appear in the aggregation process. Note that $\sum_{j=1}^{\infty} w_{j}=1$. By using the IMOWAD operator we get the infinitary IMOWAD ( $\infty$-IMOWAD) operator as follows.

$$
f\left(\left\langle u_{1}, x_{1}, y_{1}\right\rangle, \ldots,\left\langle u_{n}, x_{n}, y_{n}\right\rangle\right)=\left(\sum_{j=1}^{\infty} w_{j} b_{j}^{\lambda}\right)^{1 / \lambda}
$$

However, note that the reordering process is much more complex due to the fact that we never know which argument should go in the first or in the last position because we have an unlimited number of arguments. This problem can be partially solved by using some kind of special instructions in the order inducing variables such that we aggregate the information partially in order to obtain partial results. Further reading for the usual OWA, see. ${ }^{36}$

Note that a similar extension could be developed by using the IGMD operator, obtaining the $\infty$-IGMD operator.

A further interesting issue is the problem of ties in the reordering process of the order inducing variables. In order to solve this problem, we recommend to follow the policy explained in Ref. 21 about replacing the tied arguments by their average. Note that in this case, it would mean that we are replacing the tied arguments by their normalized Minkowski distance.

Note that in the analysis of the order-inducing variables of the IMOWAD operator, we should note that the values used can be drawn from any space, with the only requirement of having a linear ordering. Therefore, it is possible to use different kinds of attributes for the order-inducing variables that permit us, for example, to mix numbers with words in the aggregations.

Other factors that we can consider are the measures for characterizing a weighting vector and the type of aggregation it performs. ${ }^{8,15}$ The first measure $\alpha(W)$, the degree of orness, is defined as:

$$
\alpha(W)=\left(\sum_{j=1}^{n}\left(\frac{n-j}{n-1}\right)^{\lambda} w_{j}^{*}\right)^{1 / \lambda}
$$

Note that $w_{j}^{*}$ is the $w_{j}$ weight of the IMOWAD aggregation ordered in descending order according to the values of the arguments $\left|x_{i}-y_{i}\right|$. It can be shown that $\alpha \in[0,1]$. The more weight is located near the top of $W$, the closer $\alpha$ is to 1 , while the more weight is located toward the bottom of $W$, the closer $\alpha$ is to 0 . Note also that we can use the dual, that is: Andness $=1$ - Orness.

The second measure ${ }^{15}$ is called the entropy of dispersion of the weighting vector $W$. It is defined as:

$$
H(W)=-\sum_{j=1}^{n} w_{j} \ln \left(w_{j}\right)
$$

For example, if $w_{j}=1$ for some $j$, then $H(W)=0$, and thus the least amount of information is used.

The divergence of $W$ measures the divergence of the weights against the degree of orness: 


$$
\operatorname{Div}(W)=\sum_{j=1}^{n} w_{j}\left(\frac{n-j}{n-1}-\alpha(W)\right)^{2}
$$

The balance operator measures the balance of the weights against the orness or the andness:

$$
\operatorname{Bal}(W)=\sum_{j=1}^{n}\left(\frac{n+1-2 j}{n-1}\right) w_{j}
$$

It can be shown that $\operatorname{Bal}(W) \in[-1,1]$. Note that for the optimistic criteria, $\operatorname{Bal}(W)=1$, and for the pessimistic criteria, $\operatorname{Bal}(W)=-1$.

\section{Families of IMOWAD Operators}

In this Section, we analyze different particular cases of the IMOWAD operator. We distinguish between those families found in the parameter $\lambda$ and those found in the weighting vector $W$.

\subsection{Analysing the parameter $\lambda$}

By looking to the parameter $\lambda$, we can find a wide range of distance measures such as the IOWAD, the EIOWAD, the induced ordered weighted geometric distance (IOWGD) operator, the induced ordered weighted harmonic averaging distance (IOWHAD) operator and a lot of other cases.

Remark 5. When $\lambda=1$, we get the IOWAD operator.

$$
f\left(\left\langle u_{1}, x_{1}, y_{1}\right\rangle, \ldots,\left\langle u_{n}, x_{n}, y_{n}\right\rangle\right)=\sum_{j=1}^{n} w_{j} b_{j}
$$

Note that if $w_{j}=1 / n$, for all $a_{i}$, we get the NHD. The WHD is obtained if $u_{i}>u_{i+1}$, for all $i$, and the OWAD operator is obtained if the ordered position of $u_{i}$ is the same than the ordered position of $b_{j}$ such that $b_{j}$ is the jth largest of $\left|x_{i}-y_{i}\right|$.

Remark 6. When $\lambda=2$, we get the IEOWAD operator.

$$
f\left(\left\langle u_{1}, x_{1}, y_{1}\right\rangle, \ldots,\left\langle u_{n}, x_{n}, y_{n}\right\rangle\right)=\left(\sum_{j=1}^{n} w_{j} b_{j}^{2}\right)^{1 / 2}
$$

If $w_{j}=1 / n$, for all $a_{i}$, we get the NED. If $u_{i}>u_{i+1}$, for all $i$, we get the WED and if the ordered position of $u_{i}$ is the same than the ordered position of $b_{j}$ such that $b_{j}$ is the $j$ th largest of $\left|x_{i}-y_{i}\right|^{2}$, we get the EOWAD operator.

Remark 7. When $\lambda=0$, we get the IOWGD operator.

$$
f\left(\left\langle u_{1}, x_{1}, y_{1}\right\rangle, \ldots,\left\langle u_{n}, x_{n}, y_{n}\right\rangle\right)=\prod_{j=1}^{n} b_{j}{ }^{w_{j}}
$$

Note that if $w_{j}=1 / n$, for all $a_{i}$, we get the normalized geometric distance and if $u_{i}>u_{i+1}$, for all $i$, the weighted geometric distance. If the ordered position of $u_{i}$ is the same than the ordered position of $b_{j}$ such that $b_{j}$ is the $j$ th largest of $\left|x_{i}-y_{i}\right|^{w j}$, we get the ordered weighted geometric distance operator (OWGD) operator.

Remark 8. When $\lambda=-1$, we get the IOWHAD operator.

$$
f\left(\left\langle u_{1}, x_{1}, y_{1}\right\rangle, \ldots,\left\langle u_{n}, x_{n}, y_{n}\right\rangle\right)=\frac{1}{\sum_{j=1}^{n} \frac{w_{j}}{b_{j}}}
$$

Note that if $w_{j}=1 / n$, for all $a_{i}$, we get the normalized harmonic distance. If $u_{i}>u_{i+1}$, for all $i$, we get the weighted harmonic distance. If the ordered position of $u_{i}$ is the same than the ordered position of $b_{j}$ such that $b_{j}$ is the $j$ th largest of $1 /\left(w_{j} /\left|x_{i}-y_{i}\right|\right)$, we get the ordered weighted harmonic averaging distance operator (OWHAD) operator .

Remark 9. When $\lambda=3$, we get the induced ordered weighted cubic averaging distance (IOWCAD) operator.

$$
f\left(\left\langle u_{1}, x_{1}, y_{1}\right\rangle, \ldots,\left\langle u_{n}, x_{n}, y_{n}\right\rangle\right)=\left(\sum_{j=1}^{n} w_{j} b_{j}^{3}\right)^{1 / 3}
$$

If $w_{j}=1 / n$, for all $a_{i}$, we get the normalized cubic distance (NCD). If $u_{i}>u_{i+1}$, for all $i$, we get the weighted cubic distance (WCD) and if the ordered position of $u_{i}$ is the same than the ordered position of $b_{j}$ such that $b_{j}$ is the $j$ th largest of $\left|x_{i}-y_{i}\right|^{2}$, we get the ordered weighted cubic averaging distance (OWCAD) operator.

Note that we could analyze other families by using different values in the parameter $\lambda$. Note also that it is possible to study these families individually in a similar way as it has been developed in Section 3. 


\subsection{Analysing the weighting vector $W$}

By choosing a different manifestation of the weighting vector in the IMOWAD operator, we are able to obtain different types of distance aggregation operators. For example, we can obtain the maximum distance, the minimum distance, the NMD, the WMD and the MOWAD operator.

- The maximum distance is obtained if $w_{p}=1$ and $w_{j}$ $=0$, for all $j \neq p$, and $u_{p}=\operatorname{Max}\left\{\left|x_{i}-y_{i}\right|\right\}$.

- The minimum distance is obtained if $w_{p}=1$ and $w_{j}=$ 0 , for all $j \neq p$, and $u_{p}=\operatorname{Min}\left\{\left|x_{i}-y_{i}\right|\right\}$.

- More generally, if $w_{k}=1$ and $w_{j}=0$, for all $j \neq k$, we are using the step-IMOWAD operator.

- The NMD is found when $w_{j}=1 / n$, for all $i$.

- The WMD is obtained if $u_{i}>u_{i+1}$, for all $i$.

- The MOWAD operator is obtained if the ordered position of $u_{i}$ is the same than the ordered position of $b_{j}$ such that $b_{j}$ is the $j$ th largest of $\left|x_{i}-y_{i}\right|$.

- Note that the IGOWA operator ${ }^{12}$ is also included as a particular case of IMOWAD operator. This situation appears when one of the sets of the IMOWAD operator is empty.

Remark 10. Other families of IMOWAD operators could be used. For more information, see. ${ }^{8,16,19}$ For example, when $w_{j}=1 / m$ for $k \leq j \leq k+m-1$ and $w_{j}=0$ for $j>k+m$ and $j<k$, we are using the windowIMOWAD operator. Note that $k$ and $m$ must be positive integers such that $k+m-1 \leq n$.

Remark 11. If $w_{1}=w_{n}=0$, and for all others $w_{j}=1 /(n-$ 2 ), we are using the olympic-IMOWAD. Note that it is possible to present a general form of the olympicIMOWAD operator considering that $w_{j}=0$ for $j=1,2$, $\ldots, k, n, n-1, \ldots, n-k+1$; and for all others $w_{j^{*}}=1 /(n$ $-2 k$ ), where $k<n / 2$. Note that if $k=1$, then, this general form becomes the usual olympic-IMOWAD.

Remark 12. Note that the IMOWAD-median and the weighted IMOWAD-median can also be used as a particular case of the IMOWAD. For the IMOWAD median, if $n$ is odd we assign $w_{(n+1) / 2}=1$ and $w_{j}=0$ for all others, and this affects the argument $a_{i}$ with the $[(n+$ 1)/2] th largest $u_{i}$. If $n$ is even we assign for example, $w_{n / 2}=w_{(n / 2)+1}=0.5$, and this affects the arguments with the $(n / 2)$ th and $[(n / 2)+1]$ th largest $u_{i}$. For the weighted IMOWAD median, we select the argument $a_{i}$ that has the $k$ th largest inducing variable $u_{i}$, such that the sum of the weights from 1 to $k$ is equal or higher than 0.5 and the sum of the weights from 1 to $k-1$ is less than 0.5 . Note that if the ordered position of $u_{i}$ is the same than the ordered position of $b_{j}$ such that $b_{j}$ is the $j$ th largest of $a_{i}$, then, we get the IMOWAD-median and the weighted IMOWAD-median, respectively.

Remark 13. Another interesting family is the SIMOWAD operator. It can be divided in three classes, the "orlike", the "andlike" and the generalized SIMOWAD operator. The generalized S-IMOWAD operator is obtained when $w_{p}=(1 / n)(1-(\alpha+\beta))+\alpha$, with $u_{p}=\operatorname{Max}\left\{a_{i}\right\} ; w_{q}=(1 / n)(1-(\alpha+\beta))+\beta$, with $u_{q}$ $=\operatorname{Min}\left\{a_{i}\right\}$; and $w_{j}=(1 / n)(1-(\alpha+\beta))$ for all $j \neq p, q$ where $\alpha, \beta \in[0,1]$ and $\alpha+\beta \leq 1$. Note that if $\alpha=0$, the generalized S-IMOWAD operator becomes the "andlike" S-IMOWAD operator and if $\beta=0$, it becomes the "orlike" S-IMOWAD operator.

Remark 14. A further interesting family that could be used is the centered-IMOWAD operator. An IMOWAD operator is a centered aggregation operator if it is symmetric, strongly decaying and inclusive. It is symmetric if $w_{j}=w_{j+n-1}$. It is strongly decaying when $i$ $<j \leq(n+1) / 2$ then $w_{i}<w_{j}$ and when $i>j \geq(n+1) / 2$ then $w_{i}<w_{j}$. It is inclusive if $w_{j}>0$. Note that it is possible to consider a softening of the second condition by using $w_{i} \leq w_{j}$ instead of $w_{i}<w_{j}$. We shall refer to this as softly decaying centered-IMOWAD operator. Another particular situation of the centered-IMOWAD operator appears if we remove the third condition. We will refer to it as a non-inclusive centered-IMOWAD operator.

\section{The Induced Quasi-OWAD Operator}

The IMOWAD can be generalized by using quasiarithmetic means in a similar way as it was done in Ref. 12. We call it the Quasi-IOWAD operator. It is defined as follows.

Definition 7. A Quasi-IOWAD operator is a mapping $f$ : $R^{n} \times R^{n} \times R^{n} \rightarrow R$ that has an associated weighting vector $W$ with $w_{j} \in[0,1]$ and $\sum_{j=1}^{n} w_{j}=1$, such that:

$$
f\left(\left\langle u_{1}, x_{1}, y_{1}\right\rangle, \ldots,\left\langle u_{\mathrm{n}}, x_{n}, y_{n}\right\rangle\right)=g^{-1}\left(\sum_{j=1}^{n} w_{j} g\left(b_{(j)}\right)\right)
$$


where $b_{j}$ is the $\left|x_{i}-y_{i}\right|$ value of the QIOWAD triplet $\left\langle u_{i}\right.$, $\left.x_{i}, y_{i}\right\rangle$ having the jth largest $u_{i}, u_{i}$ is the order inducing variable, $\left|x_{i}-y_{i}\right|$ is the argument variable represented in the form of individual distances, and $g$ is the strictly continuous monotonic function.

As we can see, when $g(b)=b^{\lambda}$, then, the QuasiIOWAD becomes the IMOWAD operator. Note that it is also possible to distinguish between descending (Quasi-DIOWAD) and ascending (Quasi-AIOWAD) orders.

Note that all the properties and particular cases commented in the IMOWAD operator are also applicable in the Quasi-IOWAD operator.

For example, we could mention the trigonometric IOWAD operator, the exponential IOWAD operator and the radical IOWAD operator.

The trigonometric IOWAD is obtained when $g_{1}(t)=$ $\sin ((\pi / 2) t), g_{2}(t)=\cos ((\pi / 2) t)$ and $g_{3}(t)=\tan ((\pi / 2) t)$ are the generating functions. Thus, the trigonometric IOWAD functions are:

$$
\begin{aligned}
f\left(\left\langle u_{1}, x_{1}, y_{1}\right\rangle, \ldots,\left\langle u_{n}, x_{n}, y_{n}\right\rangle\right) & = \\
= & \frac{2}{\pi} \arcsin \left(\sum_{j=1}^{n} w_{j} \sin \left(\frac{\pi}{2} b_{j}\right)\right)
\end{aligned}
$$

$$
\begin{aligned}
f\left(\left\langle u_{1}, x_{1}, y_{1}\right\rangle, \ldots,\left\langle u_{n}, x_{n}, y_{n}\right\rangle\right)= & \\
= & \frac{2}{\pi} \arccos \left(\sum_{j=1}^{n} w_{j} \cos \left(\frac{\pi}{2} b_{j}\right)\right)
\end{aligned}
$$

$$
\begin{aligned}
f\left(\left\langle u_{1}, x_{1}, y_{1}\right\rangle, \ldots,\left\langle u_{n}, x_{n}, y_{n}\right\rangle\right)= \\
=\frac{2}{\pi} \arctan \left(\sum_{j=1}^{n} w_{j} \tan \left(\frac{\pi}{2} b_{j}\right)\right)
\end{aligned}
$$

The exponential IOWAD is formed when $g(t)=\gamma^{\dagger}$, if $\gamma \neq 1$, and $g(t)=t$, if $\gamma=1$. Thus, the exponential IOWAD operator is: $\log _{\gamma}\left(\sum_{j=1}^{n} w_{j} \gamma^{b_{j}}\right)$, if $\gamma \neq 1$, and the IOWAD if $\gamma=1$.

The radical IOWAD is found if $\gamma>0, \gamma \neq 1$, and the generating function is $g(t)=\gamma^{1 / t}$. Thus, the radical IOWAD operator is:

$f\left(\left\langle u_{1}, x_{1}, y_{1}\right\rangle, \ldots,\left\langle u_{n}, x_{n}, y_{n}\right\rangle\right)=$

$$
=\left(\log _{\gamma}\left(\sum_{j=1}^{n} w_{j} \gamma^{1 / b_{j}}\right)\right)^{-1}
$$

Another interesting particular type of QuasiIOWAD operator is the Quasi-weighted averaging distance (Quasi-WAD). It is found when $u_{i}>u_{i+1}$, for all $i$. It can be defined as follows.

Definition 8. A Quasi-WAD operator is a mapping $f: R^{n}$ $\times R^{n} \rightarrow R$ that has an associated weighting vector $W$ with $w_{j} \in[0,1]$ and $\sum_{j=1}^{n} w_{j}=1$, such that:

$$
f\left(\left\langle x_{1}, y_{1}\right\rangle, \ldots,\left\langle x_{n}, y_{n}\right\rangle\right)=g^{-1}\left(\sum_{j=1}^{n} w_{j} g\left(b_{(j)}\right)\right)
$$

where $b_{j}$ is the $\left|x_{i}-y_{i}\right|$ value of the $Q W A D$ tuple $\left\langle x_{i}, y_{\mathrm{i}}\right\rangle$ having the jth largest $u_{i}, u_{i}$ is the order inducing variable, $\left|x_{i}-y_{i}\right|$ is the argument variable represented in the form of individual distances, and $g$ is the strictly continuous monotonic function.

Note that if $w_{j}=1 / n$, for all $i$, then, the Quasi-WAD becomes the Quasi-normalized averaging distance (Quasi-NAD).

Note that all the properties studied in the IMOWAD and in the Quasi-IOWAD can also be studied with the Quasi-WAD operator.

\section{Numerical Example}

The IMOWAD operator can be applied in a wide range of problems including statistics, economics and engineering. In the following, we are going to develop a simple illustrative example in order to see the results obtained in the aggregation by using different types of IMOWAD operators. We develop an application in a decision making problem concerning the selection of investments where an enterprise is looking for the best strategy according to his interests.

Assume that an enterprise that operates in Europe and North America wants to invest some money the next year. In order to do so, the board of directors, after careful analysis with the group of experts of the company, has established five possible investments $S_{i}$ that the enterprise could develop in the future.

- $A_{1}$ : Invest in the Asian market.

- $A_{2}$ : Invest in the South American market. 
- $A_{3}$ : Invest in the African market.

- $A_{4}$ : Do not develop any investment.

After careful review of the information, the experts have given the following general information. They have summarized the information of the strategies in five main characteristics $C_{i}$ with the following results.

- $C_{1}$ : Risk of the investment.

- $C_{2}$ : Benefits in the short term.

- $C_{3}$ : Benefits in the long term.

- $C_{4}$ : Difficulty of the investment.

- $C_{5}$ : Other aspects.

Note that the results are valuations between 0 and 1 .

Table 1. Available investments

\begin{tabular}{cccccc}
\hline & $C_{1}$ & $C_{2}$ & $C_{3}$ & $C_{4}$ & $C_{5}$ \\
\hline$A_{1}$ & 0.7 & 0.9 & 0.8 & 0.7 & 0.3 \\
$A_{2}$ & 0.6 & 0.8 & 0.7 & 0.5 & 0.8 \\
$A_{3}$ & 0.5 & 0.6 & 0.8 & 0.4 & 0.9 \\
$A_{4}$ & 0.8 & 0.5 & 0.6 & 0.8 & 0.6 \\
\hline
\end{tabular}

According to the objectives and policies of the enterprise, the experts have established the ideal investment for the company independently of the investments available. They have established the following valuations for it.

Table 2. Characteristics of the ideal investment

\begin{tabular}{cccccc}
\hline & $C_{1}$ & $C_{2}$ & $C_{3}$ & $C_{4}$ & $C_{5}$ \\
\hline$I$ & 0.8 & 0.9 & 1 & 0.9 & 0.9 \\
\hline
\end{tabular}

In order to aggregate the information, the group of experts calculates the attitudinal character of the enterprise. Due to the fact that the attitudinal character depends upon the opinion of several members of the board of directors, it is very complex. Therefore, they need to use order inducing variables in the reordering process. The results are shown in Table 3.

Table 3. Order inducing variables

\begin{tabular}{cccccc}
\hline & $C_{1}$ & $C_{2}$ & $C_{3}$ & $C_{4}$ & $C_{5}$ \\
\hline$A_{1}$ & 12 & 16 & 20 & 24 & 8 \\
$A_{2}$ & 22 & 18 & 20 & 26 & 28 \\
$A_{3}$ & 14 & 20 & 15 & 18 & 17 \\
$A_{4}$ & 26 & 21 & 19 & 15 & 13 \\
\hline
\end{tabular}

With this information, it is possible to develop different methods for selecting an investment. In this example, we consider the NHD, the NED, the WHD, the WED, the OWAD, the IOWAD, the AIOWAD and the EIOWAD operator. Note that the weighting vector used is: $W=(0.1,0.2,0.2,0.2,0.3)$. The aggregated results obtained by using the previous particular cases of IMOWAD operators are shown in Tables 4 and 5.

Table 4. Aggregated results 1

\begin{tabular}{ccccc}
\hline & NHD & WHD & OWAD & IOWAD \\
\hline$A_{1}$ & 0.22 & 0.27 & 0.16 & 0.26 \\
$A_{2}$ & 0.22 & 0.21 & 0.19 & 0.22 \\
$A_{3}$ & 0.26 & 0.23 & 0.21 & 0.26 \\
$A_{4}$ & 0.24 & 0.27 & 0.2 & 0.27 \\
\hline
\end{tabular}

Table 5. Aggregated results 2

\begin{tabular}{ccccc}
\hline & AIOWAD & EIOWAD & Median & Olympic \\
\hline$A_{1}$ & 0.18 & 0.349 & 0.2 & 0.1 \\
$A_{2}$ & 0.22 & 0.249 & 0.2 & 0.3 \\
$A_{3}$ & 0.26 & 0.306 & 0.3 & 0.233 \\
$A_{4}$ & 0.21 & 0.305 & 0.3 & 0.3 \\
\hline
\end{tabular}

As we can see, depending on the distance aggregation operator used, the optimal choice is different. Note that the lowest value in each method is the optimal result.

If we establish an ordering of the investments, a typical situation if we want to consider more than one alternative, we will get the following orders shown in Table 6. Note that the first alternative in each ordering is the optimal choice.

Table 6. Ordering of the investments

\begin{tabular}{cc}
\hline & Ordering \\
\hline NHD & $\left.\left.A_{3}\right\} A_{4}\right\} A_{1}=A_{2}$ \\
WHD & $\left.\left.A_{1}=A_{4}\right\} A_{3}\right\} A_{2}$ \\
OWAD & $\left.\left.\left.A_{3}\right\} A_{4}\right\} A_{2}\right\} A_{1}$ \\
IOWAD & $\left.\left.\left.A_{4}\right\} A_{1}\right\} A_{3}\right\} A_{2}$ \\
AIOWAD & $\left.\left.\left.A_{3}\right\} A_{2}\right\} A_{4}\right\} A_{1}$ \\
EIOWAD & $\left.\left.\left.A_{1}\right\} A_{3}\right\} A_{4}\right\} A_{2}$ \\
Median-IOWAD & $\left.A_{3}=A_{4}\right\} A_{1}=A_{2}$ \\
Olympic-IOWAD & $\left.\left.A_{2}=A_{4}\right\} A_{3}\right\} A_{1}$ \\
\hline
\end{tabular}

As we can see, depending on the particular type of IMOWAD operator used, the results may lead to different decisions. 


\section{Conclusions}

We have presented the IMOWAD operator. It is a distance measure that uses the IOWA operator in the Minkowski distance. The main advantage of this operator is that it generalizes a wide range of distances such as the NMD, the WMD, the MOWAD, the IOWAD and the EIOWAD operator. We have studied some of its main properties.

We have further generalized the IMOWAD operator by using quasi-arithmetic means. We have called it the Quasi-IOWAD operator. We have also studied the applicability of the IMOWAD and the Quasi-IOWAD operator. We have developed an application of the new approach in a decision making problem about selection of investments. We have seen that the main advantage of using the IMOWAD and the Quasi-IOWAD is that it gives a more complete view of the decision problem.

In future research, we expect to develop further extensions of the IMOWAD and the Quasi-IOWAD operator by adding new characteristics in the problem, following the research developed in Ref. 8, and applying them to other fields.

\section{Acknowledgements}

We would like to thank the anonymous referees for valuable comments that have improved the quality of the paper. Support from the Spanish Ministry of Science and Innovation under project JC2009-00189 and the University of Barcelona under project MC238206, is gratefully acknowledged.

\section{References}

1. B.S. Ahn and H. Park, Least-squared ordered weighted averaging operator weights, Int. J. Intell. Syst. 23 (2008) 33-49.

2. G. Beliakov, A. Pradera and T. Calvo, Aggregation Functions: A Guide for Practitioners, (Springer-Verlag, Berlin, 2007).

3. F. Chiclana, E. Herrera-Viedma, F. Herrera and S. Alonso, Some induced ordered weighted averaging operators and their use for solving group decision-making problems based on fuzzy preference relations, Eur. J. Oper. Res. 182 (2007) 383-399.

4. J. Fodor, J.L. Marichal and M. Roubens, Characterization of the ordered weighted averaging operators, IEEE Trans. Fuzzy Syst. 3 (1995) 236-240.

5. J. Kacprzyk and S. Zadrozny, Towards a general and unified characterization of individual and collective choice functions under fuzzy and nonfuzzy preferences and majority via the ordered weighted average operators, Int. J. Intell. Syst. 24 (2009) 4-26.

6. N. Karayiannis, Soft learning vector quantization and clustering algorithms based on ordered weighted aggregation operators, IEEE Trans. Neural Networks 11 (2000) 1093-1105.

7. X. Liu and S. Han, Orness and parameterized RIM quantifier aggregation with OWA operators: A summary, Int. J. Approximate Reasoning 48 (2008) 77-97.

8. J.M. Merigó, New extensions to the OWA operators and their application in decision making, $\mathrm{PhD}$ Thesis, Department of Business Administration, University of Barcelona, 2008.

9. J.M. Merigó and M. Casanovas, Induced aggregation operators in the Dempster-Shafer belief structure, Int. J. Intell. Syst. 24 (2009) 934-954.

10. J.M. Merigó and M. Casanovas, The fuzzy generalized OWA operator and its application in strategic decision making, Cybernetics \& Syst. 41 (2010) 359-370.

11. J.M. Merigó and A.M. Gil-Lafuente, Using the OWA operator in the Minkowski distance, Int. J. Electrical and Computer Engin. 3 (2008) 149-157.

12. J.M. Merigó and A.M. Gil-Lafuente, The induced generalized OWA operator, Inform. Sci. 179 (2009) 729741.

13. J.M. Merigó and A.M. Gil-Lafuente, New decisionmaking techniques and their application in the selection of financial products, Inform. Sci. 180 (2010) 2085-2094.

14. Z.S. $\mathrm{Xu}$, Dependent uncertain ordered weighted averaging operators, Inform. Fusion 9 (2008) 310-316.

15. R.R. Yager, On ordered weighted averaging aggregation operators in multi-criteria decision making, IEEE Trans. Syst. Man Cybern. B 18 (1988) 183-190.

16. R.R. Yager, Families of OWA operators, Fuzzy Sets Syst. 59 (1993) 125-148.

17. R.R. Yager, Induced aggregation operators, Fuzzy Sets Syst. 137 (2003) 59-69.

18. R.R. Yager, Generalized OWA aggregation operators, Fuzzy Optim. Decision Making 3 (2004) 93-107.

19. R.R. Yager, Centered OWA operators, Soft Comp. 11 (2007) 631-639.

20. R.R. Yager, Using trapezoids for representing granular objects: Applications to learning and OWA aggregation, Inform. Sci. 178 (2008) 363-380.

21. R.R. Yager and D.P. Filev, Induced ordered weighted averaging operators, IEEE Trans. Syst. Man Cybern. B 29 (1999) 141-150.

22. R.R. Yager and J. Kacprzyk, The Ordered Weighted Averaging Operators: Theory and Applications (Kluwer Academic Publishers, Norwell, MA, 1997).

23. M. Zarghami, F. Szidarovszky and R. Ardakanian, A fuzzy-stochastic OWA model for robust multi-criteria decision making, Fuzzy Optim. Decision Making 7 (2008) 1-15.

24. S.M. Zhou, F. Chiclana, R.I. John and J.M. Garibaldi, Type-1 OWA operators for aggregating uncertain information with uncertain weights induced by type- 2 
linguistic quantifiers, Fuzzy Sets Syst. 159 (2008) 32813296.

25. D.F. Li, A ranking method of triangular intuitionistic fuzzy numbers and application in decision making, Int. J. Comp. Intelligence Syst. 3 (2010) 522-541.

26. X. Li, D. Ruan, J. Liu and Y. Xu, A linguistic-valued weighted aggregation operator to multiple attribute decision making with quantitative and qualitative information, Int. J. Comp. Intelligence Syst. 1 (2008) 274-284.

27. J.M. Merigó, Fuzzy decision making with immediate probabilities, Computers \& Industrial Engin. 58 (2010) 651-657.

28. G.W. Wei, X. Zhao and R. Lin, Some induced aggregating operators with fuzzy number intuitionistic fuzzy information and their applications to group decision making, Int. J. Comp. Intelligence Syst. 3 (2010) 84-95.

29. R.K. Brouwer, Fuzzy relational fixed point clustering, Int. J. Comp. Intelligence Syst. 2 (2009) 69-82.

30. J. Gil-Aluja, Elements for a Theory of Decision in Uncertainty, (Kluwer Academic Publishers, Dordrecht, 1999).

31. A.M. Gil-Lafuente, Fuzzy Logic in Financial Analysis, (Springer, Berlin, 2005).

32. A. Kaufmann, Introduction to the Theory of Fuzzy Subsets, (Academic Press, New York, 1975).

33. Z.S. $\mathrm{Xu}$ and J. Chen, An overview of distance and similarity measures of intuitionistic fuzzy sets, Int. J. Uncertainty Fuzziness Knowledge-Based Syst. 16 (2008) 529-555.

34. Z.S. $\mathrm{Xu}$ and J. Chen, Clustering algorithm for intuitionistic fuzzy sets, Inform. Sci. 178 (2008) 37753790.

35. R.R. Yager, New modes of OWA information fusion, Int. J. Intell. Syst. 13 (1998) 661-681.

36. R. Mesiar and E. Pap, Aggregation of infinite sequences, Inform. Sci. 178 (2008) 3557-3564.

37. R. Mesiar and J. Spirkova, Weighted means and weighting functions, Kybernetika 42 (2006) 151-160.

38. J. Spirkova, A generalization of induced Quasi-OWA operators, in: Proceedings of AGOP 2009, Mallorca, Spain, 2009, pp. 53-58.

39. V. Torra and Y. Narukawa, Some relations between Losonczi's based OWA generalizations and the ChoquetStieltjes integral, Soft Computing 14 (2010) 465-472. 